
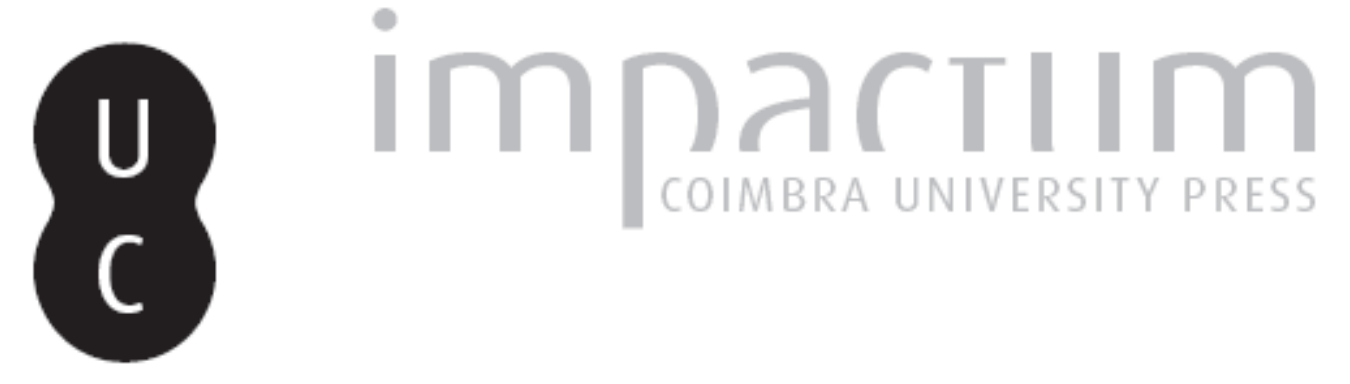

\title{
Participação em saúde: conceitos e conteúdos
}

Autor(es): $\quad$ Serapioni, Mauro; Ferreira, Pedro Lopes; Antunes, Patrícia

Publicado por: Imprensa da Universidade de Coimbra

URL persistente:

URl:http://hdl.handle.net/10316.2/35134

DOI:

DOI:http://dx.doi.org/10.14195/2183-203X_40_2

Accessed : $\quad$ 26-Apr-2023 07:27:33

A navegação consulta e descarregamento dos títulos inseridos nas Bibliotecas Digitais UC Digitalis, UC Pombalina e UC Impactum, pressupõem a aceitação plena e sem reservas dos Termos e Condições de Uso destas Bibliotecas Digitais, disponíveis em https://digitalis.uc.pt/pt-pt/termos.

Conforme exposto nos referidos Termos e Condições de Uso, o descarregamento de títulos de acesso restrito requer uma licença válida de autorização devendo o utilizador aceder ao(s) documento(s) a partir de um endereço de IP da instituição detentora da supramencionada licença.

Ao utilizador é apenas permitido o descarregamento para uso pessoal, pelo que o emprego do(s) título(s) descarregado(s) para outro fim, designadamente comercial, carece de autorização do respetivo autor ou editor da obra.

Na medida em que todas as obras da UC Digitalis se encontram protegidas pelo Código do Direito de Autor e Direitos Conexos e demais legislação aplicável, toda a cópia, parcial ou total, deste documento, nos casos em que é legalmente admitida, deverá conter ou fazer-se acompanhar por este aviso.

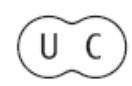




\section{Notaseco NómIcas}

LUÍS-CRUZ / PAULA SIMÕES / EDUARDQ BARATA

COMBINING OBSERVED AND CONTINGENT TRAVEL BEHAVIOUR: THE BEST OF BOTH WORLDS?

MAURO SERAPIQNI / PECDRO LOPES FERREIRA $\wedge$ PATRÍCIA ANTUNES

DOUTORAMENTO HONORIS CAUSA DO BR. ANTÓNIO ARNAUT

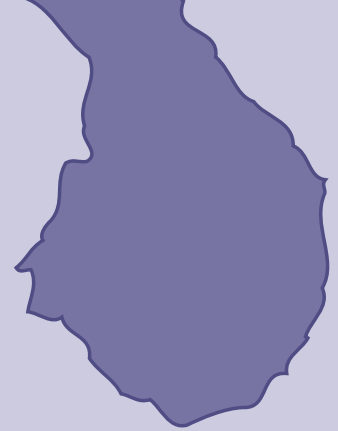

PARTICIPAÇÃO EM SAÚDE: CONCEITOS E CONTEÚDOS 


\section{résumé / abstract}

Este texto pretende contribuir para uma discussão mais aprofundada sobre as diversas formas de envolvimento dos cidadãos no sistema de saúde e para uma clarificação das várias formas de interação entre os sistemas de saúde e os cidadãos, mais concretamente, a participação, a consulta e a comunicação. Apresenta também uma breve resenha histórica das estratégias de envolvimento dos utentes na saúde, as virtudes e os desafios identificados pelos estudiosos deste setor. Por último, é apresentado o ponto da situação da participação dos cidadãos em saúde em Portugal e algumas considerações finais.

JEL Classification: I10.
Cet article entend contribuer à approfondir l'étude sur les diverses formes d'implication des citoyens dans le système de santé et à une clarification des différentes formes d'interaction entre les systèmes de santé et les citoyens, en particulier, la participation, la consultation et la communication. II comporte également un bref aperçu historique des stratégies d'implication des utilisateurs dans la santé, les vertus et les défis identifiés par les érudits de ce secteur. Finalement, il présente l'état de l'art de la participation au Portugal et quelques considérations finales.

This paper intends to contribute to further discussion about the various forms of citizens' involvement in the health system and to a clarification of the various forms of interaction between health systems and citizens, in particular, participation, consultation and communication. It also features a brief historical review of the strategies of users' involvement in health, the virtues and the challenges identified by scholars of this sector. Finally, it presents the state of the art of citizen's health participation in Portugal and some final considerations. 
Todas as organizações estão inseridas num ambiente com o qual interagem permanentemente, promovendo constantes trocas de bens e serviços. Nos serviços de utilidade pública, tais como as instituições sociais, de saúde e de educação, este intercâmbio deve ainda ser mais acentuado em consequência da forte pressão e da crescente procura de participação por parte da sociedade civil (Crouch, 2004; Santos, 2006). No caso da saúde, a resposta das organizações não tem, no entanto, sido sempre a mais adequada face à múltipla e complexa procura. A sua estrutura encontra-se ainda influenciada por uma lógica burocrática e não suficientemente orientada para a participação dos utilizadores.

A urgência em redesenhar os modelos organizacionais vigentes dentro do setor de saúde, com o propósito de aprimorar a interação com os seus beneficiários, tem caracterizado o debate político a nível internacional, especialmente desde a década de 80 do século passado. Assim, tem-se reconhecido a importância de envolver todos os atores do sistema de saúde e, ao mesmo tempo, valorizar a voz e a opinião dos utilizadores, como uma dimensão relevante no processo de produção da saúde (Giarelli, 2014; WHO, 2002). Atualmente, é consenso geral reconhecer-se que a participação representa uma estratégia essencial para melhorar a qualidade e o desempenho dos sistemas de saúde e um princípio chave a ser incorporado em todos os processos de reforma das políticas de saúde (Calnan, 1995; WHO, 2006).

Ao apresentar um enquadramento conceptual de análise do Sistema de Saúde Português, já em 2001 o Observatório Português dos Sistemas de Saúde (OPSS, 2011) destacava o papel dos cidadãos enquanto contribuintes e utilizadores e enfatizava a necessidade de se alterar consideravelmente a sua relação com o sistema de saúde. Lembrava, então, alguns direitos que, a serem exercidos, permitem situar o cidadão no centro do sistema. Entre eles importa mencionar o direito à informação, encarada como um instrumento de democratização do sistema de saúde, a par com o financiamento, a organização e a gestão dos serviços de saúde. Deixa de fazer sentido os cidadãos sentirem-se perdidos ou deambularem pelo sistema de saúde, sem orientação e sem estarem capacitados com informação adequada, de modo a poderem exercer os seus direitos.

Esta necessidade de informação passa também pelo conceito de transparência - um dos critérios mínimos de boa governação de qualquer sistema de saúde - e pelo conceito de participação. A transparência é, por exemplo, manifestada no acesso aos cuidados de saúde e na informação de que o cidadão dispõe quando espera por uma consulta ou por uma intervenção cirúrgica. A participação do cidadão no sistema de saúde engloba, por outro lado, a possibilidade de reclamação exercida através dos Gabinetes de Utente (em Portugal, desde 1986) e do Livro de Reclamações (desde 1996), o direito de ser ouvido face à opinião que tem sobre o sistema de saúde (e.g., projeto SINAS da Entidade Reguladora da Saúde) ou o impacto da sua experiência com este sistema. Passa também pela capacidade de ser envolvido nas tomadas de decisão a vários níveis do sistema, desde o nível micro da prestação direta de cuidados, ao nível macro do planeamento, da definição de prioridades e da decisão política.

Este texto pretende contribuir para uma discussão mais aprofundada sobre as diversas formas de envolvimento dos cidadãos no sistema de saúde. Na primeira parte do artigo, são apresentadas as três principais tipologias de interação entre os sistemas de saúde e os cidadãos, mais concretamente, a participação, a consulta e a comunicação. Seguidamente, o texto focaliza o tema da participação, apresentando um breve histórico das estratégias de envolvimento dos utentes na saúde, bem como as virtudes e os desafios identificados pelos estudiosos deste setor. Por último, é apresentado o ponto da situação da participação em Portugal e algumas considerações finais. 
A análise, durante os últimos trinta anos, da imensa literatura sobre experiências de interação entre sistemas de saúde e os seus beneficiários (Abelson et al., 2007; OECD, 2001; Rowe e Frewer, 2005), permite esboçar uma classificação das inúmeras iniciativas e mecanismos de envolvimento dos cidadãos nas seguintes três tipologias: (1) participação dos cidadãos; (2) consulta pública; e (3) comunicação pública. Esta classificação assenta no papel desenvolvido pelos mecanismos de participação, no nível de institucionalização e de reconhecimento social no âmbito dos respetivos sistemas de saúde, assim como na intensidade do diálogo entre representantes dos cidadãos e o sistema de saúde. Trata-se, obviamente, de três modalidades de interação muito importantes e não hierarquizáveis. São três estratégias que o sistema de saúde necessita desenvolver para poder interagir com o ambiente humano externo e, com ele, trocar sinergias, informações e recursos. Porém, como frequentemente se observa, as três formas de relação têm sido confundidas ou consideradas equivalentes. Por vezes, o levantamento da opinião dos utilizadores numa avaliação da satisfação tem sido considerada como uma praxis participativa, quando, na verdade, se trata de uma estratégia de consulta pública normalmente promovida pelos gestores de saúde com o objetivo de determinar as preferências e o grau de satisfação. Trata-se de uma importantíssima atividade para os serviços de saúde, mas que não pode ser confundida com participação.

Por esta razão, parece-nos vital propor, de acordo com a classificação de alguns autores e organizações internacionais (Abelson et al., 2007; Mitton et al., 2009; OECD, 2001; Rowe e Frewer, 2005), a classificação das tipologias de relação entre sistema de saúde e os seus utilizadores que seguidamente se analisa.

\subsection{Participação dos cidadãos}

É importante identificar os diversos níveis em que as atividades de participação dos utilizadores podem ser desenvolvidas (Charles e DeMaio, 1993; Tambuyzer et al., 2011). O primeiro nível é o nível micro, que refere a relação entre o profissional de saúde e o utente, onde escuta e participação significam construir as condições para o consentimento informado e a adesão, isto é, a participação intencional e ativa do doente nas decisões referentes à escolha de terapias e tratamentos. Esta participação transforma o doente de sujeito passivo em ator/interveniente dotado de direitos, valores e interesses. Em segundo lugar, existe um nível intermédio ou meso referente ao processo de envolvimento dos utentes dentro de um serviço ou programa específicos. Um exemplo desse tipo é a participação dos representantes dos utentes no conselho consultivo de um serviço de saúde. Este nível meso envolve a participação na definição de prioridades ou na alocação de recursos dentro de um determinado serviço. Por último, o nível macro refere a participação coletiva, quando os cidadãos são envolvidos nas decisões sobre políticas e estratégias do sistema de saúde. Neste nível, participação significa dar aos cidadãos e aos seus representantes e associações a possibilidade de influenciar, direta ou indiretamente, as possíveis opções sobre a afetação de recursos e decisões referentes à identificação de prioridades, planeamento, organização e avaliação.

Em resumo, relativamente à participação dos cidadãos, os seus mecanismos incluem duas grandes categorias de experiência de envolvimento nos sistemas de saúde. São elas a participação institucionalizada (ongoing public participation) e a participação ocasional (ad hoc public involvement)

\section{Participação institucionalizada}

Em relação à participação institucionalizada, de acordo com a literatura especializada, existem instâncias (e.g., fóruns, comités ou conselhos), formalmente reconhecidas pelas leis e normas elaboradas no âmbito dos respetivos sistemas de saúde e implementadas em todas as 
instituições de saúde da região ou do país. Exemplo histórico deste tipo de instância de participação são os Community Health Councils, instituídos em 1974 no Serviço Nacional de Saúde britânico e substituídos, em 2004, pelos Patient Forum, que por sua vez foram substituídos em 2008 pelos Local Involvement Networks (Serapioni e Romaní, 2006). Outros exemplos deste tipo de participação institucionalizada são os Citizens' Councils do Instituto Nacional de Excelência Clínica (NICE) do Reino Unido (Culyer, 2005), os Comités Consultivos Mistos da Itália (Serapioni e Duxbury, 2012), os Conselhos Municipais de Saúde do Brasil (Cotta et al., 2010) e os Conselhos da Comunidade nos Agrupamentos de Centros de Saúde (ACeS) em Portugal (OPSS, 2011). Nestes casos, a participação dos representantes dos utentes segue uma praxis participativa consolidada e integrada nas diferentes etapas do processo de gestão dos serviços de saúde. A grande maioria dos fóruns e comités mencionados, de acordo com as experiências internacionais, desenvolve um papel consultivo. Apenas numa minoria de países (e.g., Brasil, Finlândia e Nova Zelândia), os conselhos de saúde desenvolvem funções deliberativas, ou seja, participam nos processos decisionais. De reparar que, nos países anglo-saxónicos, o termo deliberativo significa a possibilidade de considerar e analisar cuidadosamente, junto com outros participantes, determinadas questões e aspetos críticos, mas não inclui necessariamente a participação direta nos processos de decisão. Nesse sentido, um grupo focal ou uma conferência pública em que os participantes podem dialogar e apresentar os seus respetivos pontos de vista são considerados espaços deliberativos. Ao contrário, os inquéritos ou outro método de recolha de informações não são definidos como abordagens deliberativas, na medida em que os entrevistados se limitam a responder a perguntas sem terem a oportunidade de discutir e argumentar coletivamente os problemas analisados.

\section{Participação ocasional}

A participação ocasional, como se pode inferir do próprio nome, diz respeito a estratégias pontuais de envolvimento dos utentes dos serviços de saúde em atividades específicas, tais como, a identificação de necessidades e de prioridades, planeamento, ou avaliação. Fala-se de estratégias ocasionais, na medida em que são iniciativas de participação esporádicas, desenvolvidas apenas em alguns distritos ou serviços de saúde e a partir de iniciativas tomadas pelas autoridades de saúde locais. Este tipo de participação é igualmente importante e necessária em determinados momentos do processo de desenvolvimento dos serviços, embora não represente uma estratégia participativa consolidada e institucionalizada. O envolvimento dos cidadãos implica, no nosso entender, um compromisso constante dos gestores e profissionais e uma cultura organizacional preparada para este tipo de interação. A consulta pública, como afirma Rigge (1995), não deve ser entendida como um exercício isolado, mas como uma componente vital para garantir a qualidade do sistema de saúde. As avaliações até agora realizadas sobre o envolvimento dos utentes têm evidenciado o valor da continuidade da participação. As experiências ocasionais podem certamente representar um instrumento efetivo, mas apenas se inseridas numa estratégia mais ampla de participação dos cidadãos (Mclver, 1998).

Exemplos deste tipo de mecanismos de envolvimento são as conferências anuais dos serviços, as assembleias públicas, os encontros no âmbito da elaboração dos planos pela saúde em Itália, os citizens' juries do Reino Unido e todos os métodos recentemente desenvolvidos nos sistemas de saúde dos países anglo-saxónicos (e.g., Reino Unido, Estados Unidos, Canadá, Austrália, Nova Zelândia) baseados nos processos deliberativos (Elster, 1998; Fishkin, 1995; Habermas, 1992), tais como as sondagens deliberativas (deliberative polls), as conferências de consenso (consensus conferences), as células de planeamento (planning cells), os grupos focais deliberativos, as oficinas dos cidadãos (citizens' workshops) e os grupos de cidadãos. Sem dúvida, os júris de cidadãos, testados pelos investigadores do Instituto de Investigação em Políticas Públicas e do King's Fund em Londres e introduzidos no sistema de saúde britânico representam a experiência de participação deliberativa mais difundida a nível internacional (Coote e Lenaghan, 1997; Parkinson, 2004). Numa fase inicial, os júris de cidadãos foram utilizados para ouvir as opiniões dos utilizadores de serviços de saúde mental e para atribuir 
financiamento aos serviços de saúde locais, mas posteriormente conquistaram popularidade ao abordar questões associadas ao racionamento dos cuidados de saúde, à definição de prioridades e à avaliação das tecnologias de saúde (Elwood e Longley, 2010; Goberman-Hill et al. 2008; Kashefi e Mort, 2004; Stafinski et al., 2012; Wakeford, 2002).

Uma outra forma de participação ocasional são, por exemplo, os processos tendentes a melhorar o envolvimento e a participação dos utilizadores idosos dos centros de saúde em Portugal (Ferreira et al., 2003; Klingenberg et al., 2005). Não prescindindo de formas mais diretas de comunicação, podem utilizar-se instrumentos especialmente desenhados para esta faixa da população e em que se pretende incentivar os doentes a escreverem, antecipadamente, as perguntas que querem fazer ao médico, de forma a melhor tirar partido do tempo em que estão na consulta (Cunningham e Newton, 2000; McKinley e Middleton, 1999) ou mesmo utilizar um pequeno diário preenchido pelo idoso e utilizado, na consulta, como forma de iniciar o diálogo médico-doente.

\subsection{Consulta pública}

A consulta pública representa uma iniciativa de envolvimento em que os responsáveis pelos serviços de saúde organizam atividades de escuta/consulta de forma a poderem recolher as opiniões e as preferências de utilizadores e cidadãos. Neste caso, os pacientes representam a fonte que alimenta o fluxo de informação a ser utilizada pelos agentes de decisão. São os organizadores do processo de consulta que decidem qual o assunto objeto da consulta e quais os sujeitos que irão fornecer as informações requeridas. Não existe, portanto, um diálogo formal (uma relação bilateral) entre os representantes do sistema de saúde e os seus utilizadores. Supõe-se que as informações reunidas representam a opinião de todos os utilizadores sobre cada assunto específico. Exemplos deste tipo de mecanismos de envolvimento são os diversos métodos para avaliar a satisfação dos utilizadores ou para aprofundar temas utilizando instrumentos como, por exemplo, questionários, entrevistas, grupos focais, painéis consultivos de cidadãos, referendos e consultas eletrónicas através de sítios interativos.

Até aos anos 80 e 90 do século passado, a própria qualidade dos cuidados prestados por uma unidade de saúde era considerada uma área da quase exclusiva competência dos profissionais de saúde, controlando estes as regras económicas com base nas quais exerciam a sua profissão, sendo escasso o relacionamento que mantinham com a sociedade. Com o final do século, porém, surge o conceito de responsabilização ou prestação de contas (accountability) associado aos profissionais, ao desempenho das organizações de saúde e, mesmo, a Governos, envolvendo também a transferência de alguns poderes para os cidadãos (Berwick et al., 1990; OPSS, 2006; UNDP, 1997; World Bank, 2003). É reconhecido, assim, o direito dos cidadãos a emitirem as suas opiniões e a serem envolvidos nos serviços de saúde (Beresford e Croft, 1993), considerado um princípio ético e, mesmo em alguns países, um requisito legal (Coulter e Elwyn, 2002).

Em Portugal, quer nos cuidados primários quer nos cuidados hospitalares, tem-se mantido uma preocupação, embora de uma forma não sistemática, de auscultação das opiniões dos cidadãos utilizadores de cuidados de saúde. Exemplos disso são as várias implementações do sistema IAQH (Instrumentos de Avaliação da Qualidade Hospitalar) que tem permitido, de uma forma anónima e estatisticamente representativa, a monitorização da satisfação, em especial, dos utilizadores dos serviços de urgência e de internamento (Pedro et al., 2010; Rodrigues, 2010), contribuindo também para os processos de acreditação em curso. Nos cuidados primários, por outro lado, tem sido realizado um levantamento mais exaustivo da satisfação, desde a prática exercida nos centros de saúde (Ferreira, 2000; Ferreira et al., 2001; Grol et al., 2000) às recentes unidades de saúde familiar (Ferreira et al., 2010).

Também os cidadãos, não sendo necessariamente utilizadores com experiência dos cuidados de saúde, têm sido auscultados de uma forma mais ou menos sistemática nos últimos anos. Exemplos disso são os Inquéritos Nacionais de Saúde levados a cabo pelo Instituto Nacional de 
Saúde Dr. Ricardo Jorge e pelo Instituto Nacional de Estatística (Portugal, 2005) e as análises das perceções dos portugueses sobre o sistema de saúde (Cabral e Silva, 2009).

Uma outra abordagem é protagonizada pelo conceito de resposta (responsiveness), que se preocupa com a avaliação de todo o sistema de saúde e com a experiência que os indivíduos têm face à adequação dos cuidados de saúde às suas legítimas expectativas, para questões não diretamente relacionadas com os níveis de saúde, mas referentes à organização dos serviços e à cultura organizacional subjacente (De Silva e Valentine, 2000). Para este conceito, amplamente divulgado pela Organização Mundial de Saúde (OMS) no seu Relatório Anual (WHO, 2000), os cidadãos são encarados na dupla qualidade de indivíduos com direitos e de utilizadores dos serviços fornecidos pelo sistema de saúde. Incluem-se, assim, áreas de respeito pelas pessoas (respeito pela dignidade, confidencialidade, direito à informação e autonomia na participação em escolhas relacionadas com a saúde) e áreas que os utentes têm em comum com qualquer utilizador (prontidão em caso de emergência e espera razoável em situações não emergentes, amenidades de qualidade adequada, acesso a redes de apoio social na família e amigos, escolha de prestador e liberdade de seleção da organização de cuidados).

Esta preocupação pela qualidade dos cuidados tem também sido encarada por quem olha para o sistema de saúde como tendo a missão de acrescentar valor aos cidadãos que a ele recorrem. É o caso da abordagem de gestão protagonizada por Michael Porter, que defende que a melhoria dos resultados em saúde e a eficiência desses cuidados, embora necessários, não são suficientes. O sistema tem também de estar desenhado em torno do conceito de valor para os cidadãos utilizadores, pois o que, em essência, se pretende não é melhorar os cuidados de saúde, mas sim melhorar a saúde dos cidadãos (Porter, 2010; Porter e Teisberg, 2006). Segundo estes autores, só assim se responde às necessidades dos cidadãos e à realidade económica atual. E, como só os próprios cidadãos são competentes para avaliar como se sentem e para contribuir para a medição do valor acrescentado pelos cuidados de saúde, as modalidades de consulta são aqui fundamentais.

\subsection{Comunicação pública}

Por último, no que respeita à comunicação pública, o fluxo informativo é unidirecional, isto é, das instituições e serviços de saúde para os cidadãos, não estando, geralmente, previsto nenhum tipo de envolvimento dos beneficiários dos serviços. Exemplos deste tipo de interação são as publicações informativas dos serviços, as campanhas de saúde, as cartas dos serviços e dos direitos e deveres dos utentes, as lojas do cidadão, as linhas telefónicas dedicadas e outras formas de "e-health" (e.g., telessaúde, telemedicina). A informação fornecida deve, no entanto, ser precisa e compreensível em relação ao valor acrescentado relativo aos resultados esperados e observados dos serviços de saúde.

Muitos estudos epidemiológicos têm demonstrado a importância da adoção de comportamentos saudáveis para melhorar a saúde da população. Estima-se que metade dos óbitos em cada ano depende de comportamentos evitáveis e de fatores sociais tais como, entre outros, o tabagismo, a obesidade e o alcoolismo (McGinnis e Froege, 1993). Outros estudos, especialmente na área da psicologia, da comunicação e da sociologia têm mostrado que a comunicação, o marketing e a informação em saúde são efetivos para induzir mudança nos estilos de vida bem como para reduzir os índices de mortalidade e de morbilidade (Evans et al., 2009; Neuhaser e Kreps, 2003). Assim, a partir dos anos 80 , foram realizadas várias intervenções com o intuito de incentivar comportamentos saudáveis, entre as quais as campanhas tradicionais de comunicação que tinham preocupações relativas à divulgação de mensagens de especialistas para o público. No entanto, vários estudos demonstraram que muitas campanhas e programas de comunicação não surtiram o efeito desejado (Kreps, 2002; Snyder et al., 2004). Esta constatação levou a questionar os modelos de comunicação adotados: os que reivindicam a capacidade de prever e explicar os comportamentos individuais face à saúde (Neuhaser e Kreps, 2003) e os que se centram na perspetiva do indivíduo como único decisor, sem considerar adequadamente a influência do 
contexto social mais amplo (Ferguson, 2002). Mais recentemente, no âmbito da psicologia social tem vindo a ser desenvolvida uma abordagem que valoriza as perceções interpessoais e intergrupos, as motivações, as normas e os fatores contextuais que influenciam os processos de comunicação. Assim, a comunicação em saúde deve adaptar-se quer às características do doente, tais como a identidade cultural, a idade e o tipo de doença (crónica ou aguda), quer ao seu contexto social e cultural e às suas representações e experiências de saúde, doença e sofrimento. Como afirmam Jones e Watson (2009:117) uma "comunicação competente no contexto da saúde é mais que a troca de informações" e deve, portanto, adaptar-se à situação do doente. Nos últimos anos, foram desenvolvidas algumas abordagens inovadoras; no entanto, os sistemas de saúde não prestam ainda atenção suficiente à forma como a informação é recebida pelos utilizadores.

É nesta ótica que faz também sentido falar de literacia em saúde, conceito definido em 1998 pela OMS como o conjunto das competências cognitivas e sociais e a capacidade dos indivíduos para acederem à compreensão e ao uso da informação de forma a promover e manter uma boa saúde (WHO, 1998), atualizada mais tarde por Kickbusch et al. (2006) como a capacidade para tomar decisões fundamentadas, no decurso da vida do dia-a-dia, em casa, na comunidade, no local de trabalho, na utilização de serviços de saúde, no mercado e no contexto político. Com esta abordagem, literacia em saúde passa a poder ser considerada como parte integrante de uma estratégia de capacitação (empowerment) dos cidadãos para aumentarem o controlo sobre a sua saúde, procurarem informação e assumirem as suas responsabilidades.

Segundo o que é defendido já há algum tempo, no atual estado de desenvolvimento do sistema de saúde português seria importante que se estabelecesse uma informação mínima a que o cidadão tivesse direito (OPSS, 2002). Isto poderia, por exemplo, incluir informação genérica sobre doenças mais vulgares e em relação às quais há algum espaço de intervenção do cidadão para as evitar ou diminuir o seu impacto, sobre sinais de risco e alerta, sobre o plano aprovado de vacinação, sobre medicamentos e farmácias e sobre hábitos propiciadores de uma vida saudável (OECD, 2001).

\section{Origem e desenvolvimento da participação dos cidadãos em saúde}

A participação comunitária nasceu da preocupação para melhorar as condições de vida das comunidades das zonas rurais dos países menos desenvolvidos do mundo (Miguel, 1986), tendo-se tornado, nos anos 50 e 60 do século passado, um tema dominante em todos os projetos de desenvolvimento rural e dos bairros marginalizados das grandes cidades. No entanto, a ideia da participação da comunidade, no âmbito da saúde, difundiu-se em todos os países do Terceiro Mundo somente no início da década de 70 . Nesta época, existia a convicção, de acordo com Zakus e Lisack (1998), de que as necessidades básicas de saúde dos países pobres podiam ser resolvidas envolvendo as populações locais. Por outras palavras, a participação das comunidades locais era considerada a estratégia principal para aumentar a disponibilidade de serviços de baixo custo para os setores mais pobres da população (Roux, 1993). Sucessivamente, a participação comunitária foi oficialmente legitimada pela OMS em 1978 durante a Conferência de Alma Ata sobre Cuidados de Saúde Primários, tendo a importância da participação da população sido reiterada em 1979 como uma estratégia fundamental para alcançar a "Saúde para Todos até ao Ano 2000".

Entretanto, diversos processos e movimentos sociais, surgidos nas sociedades ocidentais no final dos anos 60, alcançaram maturidade e provocaram efeitos significativos na saúde e na organização dos serviços. Questionar o paradigma biomédico teve como consequência o desenvolvimento de novas áreas de interesse e de investigação no âmbito da saúde: medicina familiar, comunidades terapêuticas, autoterapias e grupos de autoajuda. Neste prisma, importa enfatizar o importante papel desenvolvido pelas associações de doentes e movimentos de defesa dos direitos dos utentes, a partir dos anos 70 do século XX, que sem dúvida têm contribuído para a valorização do conhecimento leigo (lay expertise) e para pôr em causa a chamada "dominância médica" (Elston, 1991; Freidson, 1970). No mesmo período, sobretudo durante os anos 80, a 
promoção da saúde assume cada vez maior importância como estratégia para o desenvolvimento de uma nova política de saúde pública, capaz de produzir mudanças nos estilos de vida e na prevenção dos riscos ambientais (Brown, 1991; Brownlea, 1987).

Estas mudanças socioculturais dão maior relevo à importância do envolvimento dos cidadãos e à preocupação para reequilibrar a relação entre oferta e procura dos serviços de saúde. Neste sentido, a Carta de Ljubljana, assinada por todos os países europeus membros da OMS, declara, nos seus princípios fundamentais, que as reformas do setor da saúde devem "garantir que a voz dos cidadãos e suas preferências exercem uma influência determinante na forma segundo a qual os serviços são desenhados e implementados" (BMJ, 1996). A OMS tem reiteradamente apoiado esta estratégia, enfatizando e promovendo a participação dos cidadãos em todos os documentos publicados nos últimos anos (WHO, 2002, 2006). Como consequência, a participação e a capacitação dos cidadãos tornaram-se palavras-chave nos documentos de reforma da maioria dos países.

\section{A situação da participação em saúde em Portugal}

Em Portugal, a participação do cidadão é considerada um marco importante do Estado de direito democrático, sendo este, para a Constituição Portuguesa (CRP), no seu art. ${ }^{\circ} 2$, “... baseado na soberania popular, no pluralismo de expressão e organização política democráticas, no respeito e na garantia de efetivação dos direitos e liberdades fundamentais e na separação e interdependência de poderes, visando a realização da democracia económica, social e cultural e o aprofundamento da democracia participativa" (Portugal, 1976). Também a Lei de Bases da Saúde (Lei $n^{\circ} 48 / 90$, de 24 de agosto) e o Estatuto do Serviço Nacional de Saúde SNS (Decreto-Lei ${ }^{\circ} 11 / 93$, de 15 de janeiro) preveem instâncias de participação dos cidadãos na saúde, das quais se pode realçar as seguintes:

- A nível central, a Base VII da Lei de Bases da Saúde prevê a existência de um Conselho Nacional de Saúde, órgão de consulta do Governo, incluindo representantes dos utentes eleitos pela Assembleia da República.

- A nível regional, estão previstos, no art. ${ }^{\circ} 10$ do Estatuto do SNS, os Conselhos Regionais de Saúde, órgãos consultivos das respetivas Administrações Regionais de Saúde (ARS), de que fazem parte representantes dos municípios.

- A nível sub-regional (concelhio), a Lei de Bases da Saúde prevê ainda na sua Base XXVI a existência de uma Comissão Concelhia de Saúde, cuja composição é definida pelo art. ${ }^{\circ} 11^{\circ}$ dos Estatutos do SNS e integra um representante do município e um representante dos interesses dos utentes eleito pela Assembleia Municipal.

- A nível dos hospitais, foram inicialmente previstos os Conselhos Gerais (Decreto Regulamentar n. ${ }^{\circ} 3 / 88$, de 22 de janeiro, revogado pelo art. ${ }^{\circ} 42^{\circ}$ do $D L n{ }^{\circ} 188 / 2003$, de 20 de agosto), órgãos de participação e consulta que incluíam, entre outros, um representante de cada uma das Assembleias Municipais dos quatro concelhos onde residia o maior número de doentes internados no hospital durante o ano civil anterior ao da designação e um representante da associação ou liga de utentes ou amigos do hospital, quando existisse. A legislação que alterou os hospitais com natureza de sociedade anónima em entidades públicas empresariais (DL n. ${ }^{\circ}$ 233/2005, de 29 de dezembro) previu também a criação do Conselho Consultivo composto, para além de outros elementos, por um representante dos utentes designado pela respetiva associação ou por equivalente estrutura de representação e cuja competência inclui a apreciação dos planos de atividade do hospital e a emissão de recomendações, com vista ao melhor funcionamento dos serviços a prestar às populações, de acordo com os recursos disponíveis.

- A nível dos cuidados primários, foram criados os Conselhos Consultivos (DL n. ${ }^{\circ}$ 157/99, de 10 de maio) com o objetivo de assegurar a participação dos cidadãos e de instituições locais, públicas e privadas. Já no âmbito da reforma dos cuidados de saúde primários, os Agrupamentos de Centros de Saúde (ACeS), criados pelo $D L n .^{\circ} 28 / 2008$, de 22 de fevereiro, alterado pelo $D L n .^{\circ}$ 
253/2012, de 27 de novembro, preveem também a constituição do Conselho da Comunidade (CC) que envolve, entre outros, um representante da associação de utentes do ACeS. Das competências deste Conselho realça-se a de alertar o diretor executivo do ACeS para factos reveladores de deficiências graves na prestação de cuidados de saúde, de propor ações de educação, promoção da saúde e de combate à doença e de dinamizar associações e redes de utentes promotoras de equipas de voluntariado.

Para melhorar a articulação entre os cuidados de saúde primários e os cidadãos, os CC são presididos por um elemento indicado pelas câmaras municipais da área de atuação do ACeS e integram, para além deste, representantes de cada município abrangido pelo ACeS, do centro distrital de segurança social, das escolas ou agrupamentos de escolas, das instituições particulares de solidariedade social, da associação de utentes do ACeS, das associações sindicais e de empregadores com assento na Comissão Permanente de Concertação Social, do hospital de referência, das equipas de voluntariado social e da Comissão de Proteção de Crianças e Jovens.

Independentemente destas estruturas de participação dos cidadãos, escasseia informação sobre os resultados efetivos da sua existência, principalmente do seu funcionamento. Parece assim ser pertinente perguntar: será que estamos a respeitar o já anteriormente referido art. ${ }^{\circ} 2^{\circ}$ da CRP?

A ilustrar esta preocupação podemos afirmar que, em 2009, a implementação dos 74 ACeS a nível nacional possibilitaria, no limite, a criação de um número igual de CC. No entanto, o início de funcionamento destes Conselhos durante o processo de reforma dos cuidados de saúde primários não aconteceu em simultâneo, tendo alcançado, até finais de 2012, níveis de resultados muito distintos. No final desse ano, ocorreu a nível nacional um processo de concentração dos ACeS e os CC existentes acompanharam essa reestruturação, obrigando a um novo começo. De facto, a atividade dos CC, ainda antes do processo de fusão dos ACeS ter ocorrido, era muito incipiente e tal realidade, em conjunto com as alterações acentuadas nas estruturas dos ACeS, condiciona ainda hoje o seu desenvolvimento e o potencial de participação do cidadão.

Também as Agências de Contratualização, criadas pelo Despacho Normativo n. ${ }^{\circ} 46 / 97$, de 8 de agosto (uma em cada região de saúde), tinham como missão constituir-se como instâncias regionais intermédias entre a sociedade e os serviços prestadores de cuidados. Isto foi materializado pelo cumprimento das linhas orientadoras da contratualização (e.g., as teorias do agente principal e a new public management) e, apenas no caso de Lisboa e Vale do Tejo, pela criação da Comissão de Acompanhamento Externo dos Serviços de Saúde, englobando nas negociações um representante da população. No entanto, com alterações de liderança do Ministério da Saúde, as Agências foram, sem qualquer explicação pública, desativadas. Quando mais tarde renasceram, já como Departamentos de Contratualização das ARS, a sua independência baseada na separação entre o prestador e o pagador foi reduzida e a sua missão ficou muito focada nos aspetos financeiros da prestação de cuidados, situação que permanece hoje em dia.

Por outro lado, o Plano Nacional de Saúde 2004-2010 atribuiu um claro ênfase à centralidade do sistema no cidadão (Portugal, 2004), tendo determinado algumas ações estratégicas, de que se destaca, por exemplo, aumentar as opções de escolha do cidadão e, consequentemente, o aumento do seu acesso a informação validada, isenta e pertinente. Este Plano reconheceu também a "pobreza de mecanismos formais e informais para encorajar a participação dos cidadãos no sistema de saúde" e propôs dar voz à cidadania através de organizações da sociedade civil.

Tal como acontece noutros países, encontramos em Portugal uma grande discrepância entre o que está estipulado na lei sob o ponto de vista normativo e a prática do nosso sistema de saúde. $\mathrm{Na}$ ausência de canais institucionais de participação, é fundamental reconhecer e fortalecer o papel e a importância das organizações de cidadãos, pela sua potencialidade de influenciar quem toma as decisões no âmbito do sistema de saúde. 
Atualmente, está a retomar-se uma discussão anterior sobre o papel das ARS no SNS, existindo opiniões que as acusam de alguma escassez de desempenho em áreas de definição de estratégias regionais e outras que consideram que é fundamental a proximidade entre a administração da justiça, as entidades prestadoras de cuidados e os cidadãos.

Considera-se, assim, importante investir em três áreas interligadas: (1) a educação ao nível dos primeiros anos de escolaridade sobre aspetos de exercício da cidadania e de educação para a saúde; (2) a auscultação regular das opiniões e perceções dos cidadãos sobre o sistema de saúde e a experiência vivida; e (3) a necessidade de se iniciarem, a um nível micro, experiências de participação e envolvimento do cidadão no sistema de saúde, de se analisarem os resultados, de corrigir o que houver a corrigir e de alargar a experiência num processo controlado.

\section{Conclusão}

Na conceção atual, a participação representa uma estratégia para recuperar a responsabilidade e a autonomia dos utentes através de uma relação mais igualitária com os profissionais, com o objetivo de contribuir para o aumento da difusão da informação na população e para dar voz aos utilizadores sobre aspetos essenciais da saúde e da organização dos serviços. Além disso, torna-se importante considerar, tal como assinalam diversos autores (Barnes et al., 2008; Rhodes e Nocon, 1998), que os cidadãos e os utilizadores dos serviços, graças à sua experiência pessoal e conhecimento (perícia), possuem uma perspetiva privilegiada que Ihes permite participar na formulação de políticas, no desenho dos serviços e na definição dos resultados esperados (Bovemkamp et al., 2010; Weiss, 1986; Williams, 1994). Esta posição é, muitas vezes, acompanhada pelos próprios doentes, quando esperam ser envolvidos, sendo esse envolvimento uma das responsabilidades dos médicos e restantes prestadores de cuidados de saúde (Buetow, 1998; Verhoef et al., 1999).

A análise da literatura sobre participação em saúde permite observar que, a nível internacional, há um amplo consenso sobre a importância do envolvimento dos cidadãos em distintos momentos do processo de produção da saúde. De entre algumas das inúmeras virtudes e vantagens identificadas pelos estudiosos da participação em saúde pode destacar-se a melhoria e o aumento da aceitabilidade das decisões, a melhoria da comunicação entre o sistema de saúde e os cidadãos, o reequilíbrio da relação entre oferta e procura, a valorização da responsabilidade e autonomia do cidadão, a contribuição para a definição de políticas de saúde e de prioridades, o favorecimento do envolvimento nas atividades de promoção da saúde, a legitimidade das decisões sobre questões complexas referentes tanto à avaliação custo-efetividade de determinadas intervenções como aos dilemas éticos colocados pelas inovações tecnológicas, e o reconhecimento do importante papel das associações de voluntariado que desenvolvem crescentes atividades de cuidado informal.

Simultaneamente, a literatura analisada alerta para a dificuldade de promover e implementar a prática da participação. Nesse sentido, os resultados de inúmeras investigações indicam que, apesar das boas intenções e de alguns esforços louváveis empreendidos, a participação ainda tarda em entrar na praxis dos serviços de saúde (Crawford et al., 2002; Rowe e Frewer, 2004). Nesse sentido, muitos investigadores registaram a contradição entre um discurso sobre o envolvimento que realça o processo de reforma dos sistemas de saúde e uma realidade que mostra como a participação ainda demora a entrar na praxis dos serviços de saúde (Zackus e Lisack, 1998). Um ponto crítico assinalado pela literatura diz respeito à ausência de processos de avaliação das experiências de participação no âmbito dos serviços de saúde, que demonstrem a efetividade e a qualidade das atividades participativas implementadas (Burton, 2009; WHO, 2006). Neste sentido, as reflexões de muitos investigadores convergem na preocupação de definir o conceito de 'efetividade' da participação pública e consequentemente identificar critérios idóneos para a avaliar (Serapioni e Matos, 2013). Na revisão da literatura sobre o tema da avaliação da participação efetuada por Rowe e Frewer (2004), por exemplo, cerca de metade dos estudos publicados adotaram critérios de outcome (resultado) e a outra metade critérios baseados no processo. 
Outro tema, já amplamente analisado pela investigação realizada nos anos 90 , é o da representatividade das associações de doentes, que nem sempre conseguem ser garante da identificação das necessidades dos setores mais vulneráveis da sociedade (Contandriopoulos, 2004; Frankrish et al., 2002). Finalmente, um tema emergente em muitos artigos diz respeito às estratégias e aos métodos adotados para envolver doentes e cidadãos. Na perspetiva da governança, nos últimos anos obtiveram muito êxito as diversas formas de democracia deliberativa (face-to-face discussion), com vista a reforçarem a interação bidirecional entre utilizadores e gestores (Abelson et al., 2003; Delli Carpini et al., 2004).

Concluindo, os resultados destas reflexões evidenciam uma situação dinâmica e ainda em fase de evolução dos sistemas de saúde. Neste sentido, são visíveis as mudanças na organização dos serviços de saúde, como resultado da crescente necessidade de controlo da qualidade por parte dos utilizadores e da maior exigência de personalização e humanização dos cuidados.

Os processos de mudança obviamente afetam todos os atores envolvidos na área da saúde, levando a uma nova fase em que começam a ser experimentadas e desenvolvidas novas relações entre o sistema de saúde, de uma parte, e os doentes, os cidadãos e as organizações da sociedade civil, de outra.

\section{Referências}

Abelson, J.; Forest, P.G.; Eyles, J.; Smith, P.; Martin, E.; Gauvin, F.P. (2003) Deliberations about deliberative methods: Issues in the design and evaluation of public participation process, Social Science and Medicine, 57, 239-251.

Abelson, J.; Giacomini, M.; Lehoux, P.; Gauvin, F.-P. (2007) Bringing the public into health technology assessment and coverage policy decision: From principles to practice, Health Policy, 82, 37-50.

Barnes, M.; Skelcher, C.; Beirens, H.; Daiziel, R.; Jeffares, S.; Wilson. L. (2008) Designing citizen-centred governance, Birmingham, Joseph Rowentree Foundation.

Beresford, P.; Croft, S. (1993) Citizen involvement: A practical guide for change, London: Macmillan.

Berwick, D.M.; Godfrey, A.B.; Roessner, J. (1990) Curing Health Care, San Francisco, Jossey-Bass. BMJ (1996) The Ljubljana Charter on reforming healthcare, British Medical Journal, 312, 1664-1665.

Bovenkamp, H. van de; Trappenburg, M. (2010) Patient participation in collective healthcare decision making: The Dutch model, Health Expectations, 13, 73-85.

Brown, E.R. (1991) Community action for health promotion: A strategy to empower individuals and communities, International Journal of Health Service, 21(3), 441-456.

Brownlea, A. (1987) Participation: Myths, realities and prognosis, Social Science and Medicine, 25(6), 605-614.

Buetow, S. (1998) The scope for the involvement of patients in their consultations with health professionals: Rights, responsibilities and preferences of patients, Journal of Medical Ethics, 24, 243-247.

Burton, P. (2009) Conceptual, theoretical and practical issues in measuring the benefits of public participation, Evaluation, 15(3), 263-284.

Cabral, M.V.; Silva, P.A. (2009) O estado da saúde em Portugal, Lisboa, ICS.

Calnan, M. (1995) Citizens' views on health care, Journal of Management in Medicine, 9, 4, 17 - 23.

Charles, C.; DeMaio, S. (1993) Lay participation in health care decision making: A conceptual framework, Journal of Health Politics, 18(4), 881-904. 
Contandriopoulos, D. (2004) A sociological perspective on public participation in health care, Social Science \& Medicine, 58, 321-330.

Coote, A.; Lenaghan, J. (1997) Citizens' juries: Theory into practice, Institute for Public Policy Research - IPPR, London.

Cotta, R.M.M.; Cazal, M.M.; Martins, P.C. (2010) Conselho Municipal de Saúde: (Re)Pensando a lacuna entre o formato institucional e o espaço de participação social, Ciência \& Saúde Coletiva, 15(5), 2437-2445.

Coulter, A.; Elwyn, G. (2002) What do patients want from high-quality general practice and how do we involve them in improvement?, British Journal of General Practice, 52, S22-S26.

Crawford, M. J.; Rutter, D.; Manley, C.; Weaver, T.; Bhui, K.; Fulop, N.; Tyrer, P. (2002) Systematic review of involving patients in the planning and development of health care, British Medical Journal, Nov 30; 325(7375), 1263.

Crouch, C. (2004) Post-Democracy, Cambridge, Polity Press, Cambridge.

Culyer, A.J. (2005) Involving stakeholders in healthcare decisions, Healthcare Quarterly, 8(2), 56-60.

Cunningham, C.; Newton, R. (2000) A question sheet to encourage written consultation questions, Quality in Health Care, 9, 42-46.

De Silva, A.; Valentine, N. (2000) Measuring responsiveness: results of a key informants survey in 35 countries, GPE Discussion Paper No. 21, Geneva, World Health Organization.

Delli Carpini, M.X.; Cook, F.M.; Jacobs, L.R. (2004) Public deliberation, discursive participation, and citizen engagement: A review of the empirical Literature, Annual Review of Political Science, 7, 314-344.

Elster, J. (1998) Deliberative Democracy, Cambridge, Cambridge University Press.

Elston, M.A. (1991) The politics of professional power: medicine in a changing health service. In: Gabe, J.; Calnan, M.; Bury, M. (orgs.), The Sociology of the Health Care Service, Routledge, London.

Elwood, P.; Longley, M. (2010) My health: Whose responsibility? A jury decides, Journal of Epidemiology and Community Health, 64(9), 761-764.

Evans, W.D.; Uhrig, J.; Davis, K.; McCormack, L. (2009) Efficacy methods to evaluate health communication marketing campaigns, Journal of Health Communication, 14, 315-330.

Ferguson, T. (2002) From patients to end users, British Medical Journal, 324, 555-556.

Ferreira, P.L. (2000) Avaliação dos doentes de cuidados primários: enquadramento conceptual, Revista Portuguesa de Clínica Geral, 16, 53-62.

Ferreira, P.L.; Antunes, P.; Portugal, S. (2010) O valor dos cuidados primários: Perspectiva dos utilizadores das USF, Lisboa, Ministério da Saúde.

Ferreira, P.L.; Luz, A.; Valente, S.; Raposo, V.; Godinho, P.; Felício, E.D. (2001) Determinantes da satisfação dos utentes dos cuidados primários: o caso de Lisboa e Vale do Tejo, Revista Portuguesa de Saúde Pública, Volume Temático 2, 53-61.

Ferreira, P.L.; Raposo, V.; Pisco, L. (2003) Envolvimento de doentes idosos em clínica geral: Instrumentos e instruções. Nijmegen: Holanda.

Fishkin, J.S. (1995) The Voice of the People. Public Opinion and democracy, New Haven and London, Yale University Press.

Frankish, C.J.; Kwan, B; Ratner, P.A.; Higgins, J.W.; Larsen, C. (2002) Challenges of citizen participation in regional health authorities, Social Science and Medicine, 54, 1471-1480.

Freidson, E. (1970) Professional dominance, Atherton, New York. 
Giarelli, G. (2014) La sfida dell'auto-mutuo aiuto. L'associazionismo di cittadinanza come fattore di coesione sociale, Praxis Sociológica, 18, 15-32.

Gooberman-Hill, R.; Horwood, J.; Calnan, M. (2008) Citizens' juries in planning research priorities: process, engagement and outcome, Health Expectation, 11, 272-281.

Grol, R.; Wensing, M.; Mainz, J.; Jung, H.P.; Ferreira, P.L.; Hearnshaw, H.; Hjortdahl, P.; Olesen, F.; Reis, S.; Ribacke, M.; Szecsenyi, J. (2000) Patients in Europe evaluate general practice care: An international comparison, British Journal of General Practice, 50(460), 882-887.

Habermas, J. (1992) Further reflections on the Public Sphere. In Calhoun, C. (Eds.), Habermas and the Public Sphere, Cambridge, Massachusetts, MIT Press.

Jones, L.; Watson, B. (2009) Complex health communication. A language and social psychological perspective, Journal of Language and Social Psychology, 28(2), 115-118.

Kashefi, E.; Mort, M. (2004) Grounded citizens' juries: A tool for health activism?, Health Expectations, 7, 290-302.

Kickbusch, I. (2006) Health promotion: Not a tree but a rhizome. In O'Neill, M.; Pederson, A.; Dupere, S.; Rootman, I. (Eds.), Health promotion in Canada: critical perspectives, Toronto, Canadian Scholars Press.

Kickbusch, I.; Wait, S.; Maag, D. (2006) Navigating health: The role of health literacy, London, Alliance for Health and the Future, International Longevity Centre-UK.

Klingenberg, A.; Hearnshaw, H.; Wensing, M.; Ferreira, P.L.; Raposo, V.; Szecsnyi, J. (2005) Older patients' involvement in their health care: Can paper-based tools help? A feasibility study in 11 European countries, Quality in Primary Care, 13, 235-242.

Kreps, G.L. (2002) Evaluating new health information technologies: expanding the frontiers of health care delivery and health promotion, Studies in Health, Technology and Informatics, 80, 205-212.

McGinnis, J.M.; Froege, W.H. (1993) Actual causes of death in the United States, Journal of the American Medical Association, 270(18), 2207-2211.

Mclver, S. (1998) Healthy debate? An independent evaluation of citizens' juries in health setting, Londres, King's Fund.

McKinley, R.K.; Middleton, J.F. (1999) What do patients want from doctors? Content analysis of written patient agendas for the consultation, British Journal of General Practice, 49, 796-800.

Miguel, J.M. (1986). La salud pública del futuro, Madrid, Ariel Sociologia.

Mitton, C.; Smith, N.; Peacock, S.; Evoy, B.; Abelson, J. (2009) Public participation in health care priority setting: A scoping review, Health Policy, 91, 219-228.

Neuhauser, L.; Kreps, G.L. (2003) Rethinking Communication in the E-health Era, Journal of Health Psychology, 8(1), 7-23.

OECD (2001) Citizens as Partners. Paris, Organization for Economic Co-operation and Development.

OPSS (2002) O estado da saúde e a saúde do Estado, Lisboa, Observatório Português dos Sistemas de Saúde.

OPSS (2006) Um ano de governação em saúde: Sentidos e significados, Coimbra, Mar da Palavra.

OPSS (2011) Da repressão da crise para a governação prospectiva da saúde, Escoval, A.; Lopes, M.; Ferreira, P.L. (coords.), Coimbra, Mar da Palavra.

Parkinson, J. (2004) Why deliberate? The encounter between deliberation and new public managers, Public Administration, 82(2), 377-395. 
Pedro, S.; Ferreira, P.L.; Mendes, J. (2010) Aplicação de métodos estatísticos na avaliação da satisfação dos utentes com o internamento hospitalar, Tourism \& Management Studies, 6, 175189.

Porter, M.E. (2010) What is value in health care?, NEJM, 326(26), 2477-2481.

Porter, M.E.; Teisberg, E. (2006) Redefining health care. Creating value-based competition on results, Boston, Harvard Business School Press.

Portugal, INSA, INE. (2005) Inquérito Nacional de Saúde, Lisboa, Instituto Nacional de Saúde.

Portugal, Ministério da Saúde, Plano Nacional de Saúde 2004-2010 (2004), Lisboa, DirecçãoGeral da Saúde.

Rhodes, P.; Nocon, A. (1998) User involvement and the NHS reforms. Health Expectations, 1(2), 73-81.

Rigge, M. (1995) Does public opinion matter?, Health Service Journal, 7: 26-27.

Rodrigues, R. (2010) Satisfação com os serviços de urgência. Dissertação de Mestrado em Gestão e Economia da Saúde, Coimbra, Faculdade de Economia da Universidade de Coimbra.

Roux, G.I. (1993) Participación y cogestión de la salud, Educación Médica y Salud, 27(1), 50-60.

Rowe, G.; Frewer, L.J. (2004) Evaluating Public-Participation Exercises: A research agenda, Science, Technology and Human Value, 29(4), 512-557.

Rowe, G.; Frewer, L. (2005) A typology of public mechanisms, Science, Technology and Human Value, 30(2), 251-290.

Santos, B.S. (2006) Para um novo senso comum. A ciência, o direito e a política na transição paradigmática, Vol. 4; Porto, Edições Afrontamento.

Serapioni, M; Matos, A.R (2013) Participação em saúde: Entre limites e desafios, rumos e estratégias, Revista Portuguesa de Saúde Pública, 31(1), 11-22.

Serapioni, M.; Duxbury, N. (2012) Citizens participation in the Italian health-care system: the experience of the Mixed Advisory Committees, Health Expectations, Apr 19.

Serapioni, M.; Romaní, O. (2006) Potencialidades e desafios da participação em instâncias colegiadas dos sistemas de saúde: Os casos de Itália, Inglaterra e Brasil, Cadernos de Saúde Pública, 22, 11, 2411-2421.

Snyder, L.B.; Hamilton, M.A.; Mitchell, E.W.; Kiwanuka-Tondo, J.; Fleming-Milici, F.; Proctor, D. (2004) A meta-analysis of the effect of mediated health communication campaigns on behavior change in the United States, Journal of Health Communication, 9(1), 71-96.

Stafinski, T.; Menon, D.; Yasiu, Y. (2012) Assessing the impact of deliberative processes on the views of participants: Is it 'in one ear and out the other'?, Health Expectations, 17, 278-290.

Tambuyzer, E.; Pieters, G.; Van Audenhoven, V. (2011) Patient involvement in mental health care: One size does not fit all, Health Expectations, 17, 138-150.

UNDP (1997) Governance for sustainable human development: A UNDP policy document, New York, United Nations Development Programme.

Verhoef, M.J.; White, M.A.; Doll, R. (1999) Cancer patients' expectations of the role of family physicians in communication about complementary therapies, Cancer Prevention and Control, 3, 181-187.

Wakeford, T. (2002) Citizens Juries: A radical alternative for social research, Social Research Update, 37, 1-5.

Weiss, S.J. (1986) Consensual norms regarding patient involvement, Social Science \& Medicine, 22, 489-496. 
WHO (1998) Health promotion glossary, Geneva, World Health Organization.

WHO (2000) Health Report 2000, Geneva, World Health Organization.

WHO (2002) Community participation in local health care and sustainable development: approaches and techniques, Geneva, World Health Organization.

WHO (2006) Ninth Future Forum on health system governance and public participation, Copenhagen, World Health Organization.

Williams, B. (1994) Patient satisfaction: a valid concept?, Social Science \& Medicine, 38, 509516.

World Bank (2003) MENA Development Report: Better governance for development in Middle East and North Africa: Enhancing inclusiveness and accountability, Washington DC, World Bank. Zakus, D.; Lysack, C. (1998) Revisiting community participation, Health Policy and Planning, 13(1), 1-12. 